\title{
Muertes bajo sospecha: Investigación sobre el número de fallecidos en el desastre del estado Vargas, Venezuela, en 1999.
}

Deaths under suspicion: Investigation on the number of deaths in the Vargas state disaster, Venezuela, in 1999.

\section{R. Altez ${ }^{1}$}

RESUMEN

El desastre ocurrido en diciembre de 1999 en el estado Vargas, Venezuela, dio la vuelta al mundo como noticia espectacular, en donde el número de fallecidos siempre osciló entre decenas de miles. Muchas de estas cifras fueron el resultado de declaraciones inexpertas o irresponsables, desde las cuales se nubló toda posibilidad de precisión al respecto, contribuyendo con ello a que la noción sobre aquel evento alimentara la imaginería y no la interpretación. Este trabajo se concentró en la sistematización de la información existente en los servicios forenses que intervinieron en el levantamiento de los cadáveres, así como en otras instancias vinculadas al caso, acompañando los razonamientos con aproximaciones cualitativas a la zona del desastre. Los resultados ofrecidos a la vuelta de esta investigación, no sólo discuten las cifras ofrecidas desde aquel momento, sino que también evidencian la problemática resultante de la no aplicación de metodologías eficaces para el trato de cadáveres en desastres con muertes masivas, demostrando, del mismo modo, la necesidad de desarrollar investigaciones formales sobre casos similares.

Palabras clave: Desastres de masas, muertes, estado Vargas, Venezuela.

Cuad Med Forense 2007; 13(50):255-268

\section{ABSTRACT}

The disaster happened in December of 1999 in the Vargas state, Venezuela, run around the world like spectacular news, in which the number of deceases always oscillated between tens of thousands. Many of these numbers were the result of inexpert or irresponsible declarations, from which all possibility of precision became cloudy on the matter, contributing with it to that the notion on that event fed suppositions and not interpretations. This work are focused in the systematization of the existing information in the forensic offices that took part in the rise of corpses, as well as other instances to the case, accompanying the reasoning with qualitative approaches to the disaster zone. The results offered around this investigation, not only discuss the numbers offered from that moment, but that also demonstrate the problem result from not application of efficient methodologies for the treatment of disasters with massive deaths, demonstrating, in the same way, the necessity to develop formal investigations on similar cases.

Key words: Mass disasters, deaths, Vargas state, Venezuela.

Correspondencia: Prof. Rogelio Altez. Escuela de Antropología. Departamento de Etnología y Antropología Social. Facultad de Ciencias Económicas y Sociales. Universidad Central de Venezuela. Teléfono: 58416703 3225. Fax: 582126052466. E-mail: ryaltez@cantv.net.

1 Antropólogo y Magister en Historia de las Américas 


\section{INTRODUCCION:}

Entre el 15 y el 17 de diciembre de 1999 Venezuela padeció uno de los mayores desastres de su historia, que se convirtió en uno de los más grandes de Latinoamérica. Una gran cantidad de regiones del país se vieron afectadas por una vaguada de tres días consecutivos, con lluvias incesantes que provocaron aludes, inundaciones y arrastres de materiales a lo largo de toda la costa caribeña. El estado Vargas (Fig. I), ubicado en una estrecha franja costera al Norte de Caracas que se desplaza entre mar y montañas con elevaciones hasta los 2.600 metros, padeció la descarga de agua más considerable del fenómeno, la cual recorrió la abrupta topografía de la zona, produciendo los aludes torrenciales de peores consecuencias al respecto. Daños materiales calculados en más de dos mil millones de dólares [I], y el cálculo acerca del número de fallecidos estimado entre 15.000 y 50.000 víctimas,

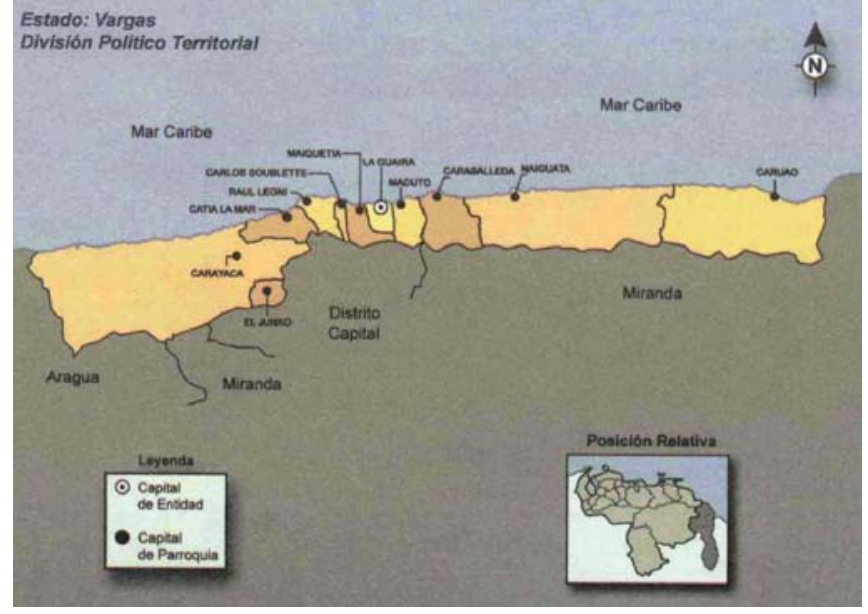

Figura 1: Situación relativa del estado Vargas, Venezuela. otorgan un protagonismo histórico al evento.

Se trató de un desastre de grandes proporciones (Fig. 2) en donde se evidenciaron también las condiciones de respuesta de la sociedad venezolana ante eventos catastróficos como este. Cuando un hecho desastroso tiene lugar, debe observarse como el resultado del cruce de dos variables fundamentales y anteriores al desenlace (amenaza y vulnerabilidad), se combinan en tiempo y espacio para desatar una gran adversidad. El mismo fenómeno natural, de haber sucedido en un contexto con otras condiciones (llanos, desiertos, o en el medio del océano), no hubiese causado

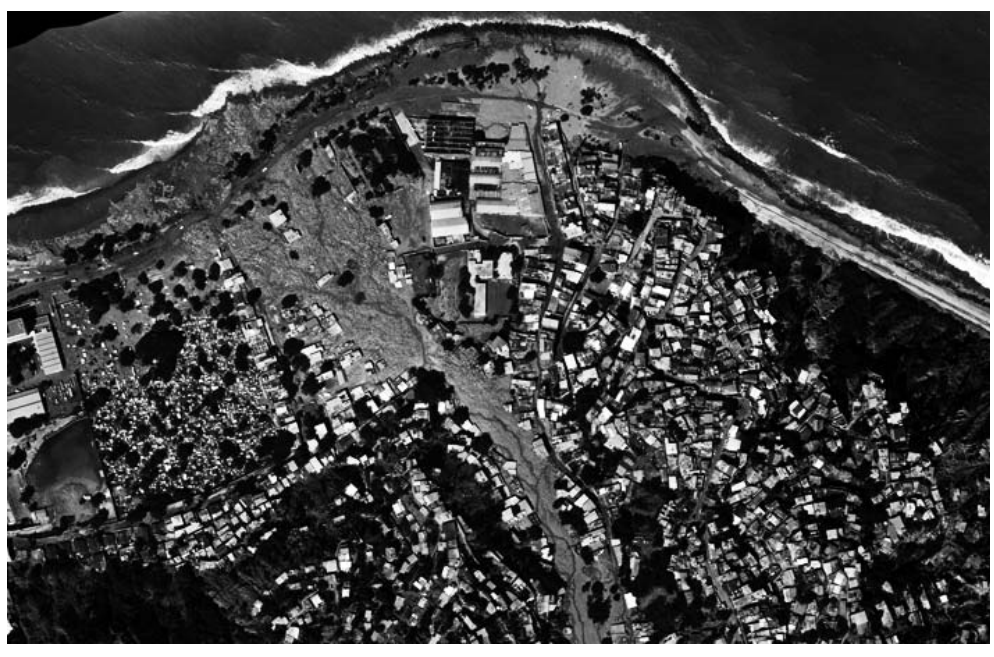

Figura 2: Ortofotografía de la zona de Punta de Mulatos, en el estado Vargas, días después de los efectos de las lluvias de diciembre de 1999. [6] las mismas consecuencias. Esto es lo que conduce a comprender que los desastres no son naturales [2,3], y que son el resultado de una construcción histórica y social $[4,5]$. No obstante, la observación de los efectos de estos eventos no debe reducirse al impacto único de las pérdidas humanas y materiales, sino que también debe apreciarse en derivaciones que contribuyen al trastorno del orden y a la 
profundización de las consecuencias negativas. Uno de estos aspectos tiene lugar, precisamente, en la intervención durante los momentos de mayor impacto y en la forma en la que posteriormente se enfrenta la reconstrucción y rehabilitación de la zona afectada.

Dentro de los aspectos que tienen que ver con la intervención inmediata, rescatar y atender a las víctimas (damnificados, desplazados y fallecidos), resulta uno de los indicadores más fehacientes de la capacidad de respuesta que una sociedad posee frente al advenimiento de un desastre. En diciembre de 1999, al igual que en otros desastres del pasado venezolano y que en otros lugares del mundo en eventos similares, se demostró claramente que para el momento no se poseían recursos técnicos o metodológicos que atendieran positivamente una tragedia de muertes masivas. Perera [7] y Morgan y cols. [8], luego de las consecuencias del tsunami del sur asiático, han hecho énfasis en la ausencia de métodos y técnicas al respecto, y en las consecuencias legales, sociales y éticas de esta carencia. Previamente, la Organización Panamericana de la Salud (OPS), en la presentación de su guía titulada Manejo de cadáveres en situaciones de desastre, afirmaba la necesidad de revisar este aspecto con mayor cuidado, calificando de "fundamental" el trato y la atención inmediata al problema, por parte de las autoridades nacionales, regionales o locales vinculadas al desastre. Como uno de los ejemplos de desastres recientes, a partir de los cuales se elaboró la citada guía, se señalaba a las inundaciones de Venezuela de 1999.

Este trabajo pretende señalar una de las consecuencias negativas que resulta de la ausencia de metodologías y sistematizaciones para la atención de los cadáveres luego de un desastre con muertes masivas, tomando como ejemplo el caso del estado Vargas en Venezuela, escenario del desastre de 1999, donde hasta el presente no se han podido establecer cifras oficiales sobre el número de víctimas ni se ha podido dar una respuesta formal al caso de los desaparecidos.

\section{SOBRE EL PROBLEMA A TRATAR:}

En medio de la confusión general, y mientras se declaraban públicamente los cálculos sobre el número de fallecidos, el levantamiento de cadáveres en el estado Vargas se llevó a cabo de manera casi inadvertida, pues los resultados de este trabajo no fueron tomados en cuenta a la hora de calcular el número total de víctimas. Al mismo tiempo, el trato a damnificados y desplazados se veía sumergido en pragmatismos y fachadas políticas, agravando las circunstancias. Toda esta escena contribuyó decididamente a pintar un panorama borroso acerca de la situación, en donde los protagonismos apresurados impulsaron declaraciones que, aún en la actualidad, dejan sentir su eco. La amplia brecha que indica la oscilación entre 15.000 y 50.000 víctimas fatales, es un indicador de la imprecisión resultante y de la ausencia de sistematización en el abordaje del caso.

Las declaraciones de las autoridades más importantes para el momento, comenzaron a dar señales de estimaciones basadas en la apreciación de las escenas a partir de la impresión causada por el caso (Fig. 3), pero no por el despliegue de técnicas apropiadas para ello. Defensa Civil declaró en un principio como "incalculable" el número de fallecidos, para luego decir que rondaba los "30.000 sepultados" [9]; poco después, George Weber, representante de Cruz Roja

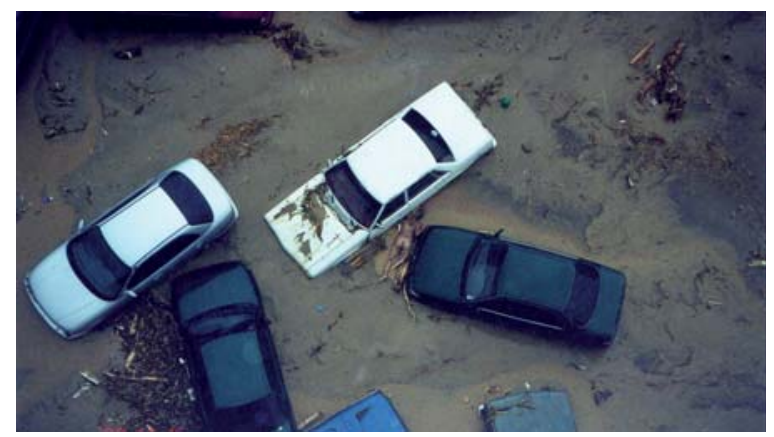

Figura 3: Imagen de uno de los cuerpos hallados en medio del desastre. Fotografía anónima. 
Internacional, aseguró que las víctimas triplicaban el número causado por el célebre huracán Mitch de Centro América y que el número debía ser el de unos 50.000 fallecidos [10]. Muchas otras acompañaron el caso, especialmente las que provenían de los medios de comunicación, responsables de difundir las declaraciones oficiales y de interpretar esas declaraciones conforme al estilo generalmente amarillista de la prensa y la televisión. Algunos de los titulares, ciertamente, resaltaban los calificativos de "Tragedia capital", "Incalculable el número de muertes", "Decenas de miles de desaparecidos", "Insuficientes urnas y fosas" (Fig. 4), con lo cual el desastre se desplegaba hacia cifras inimaginables, grabando una impronta mucho más cercana al mito y la leyenda, que a una apreciación certera de la realidad.

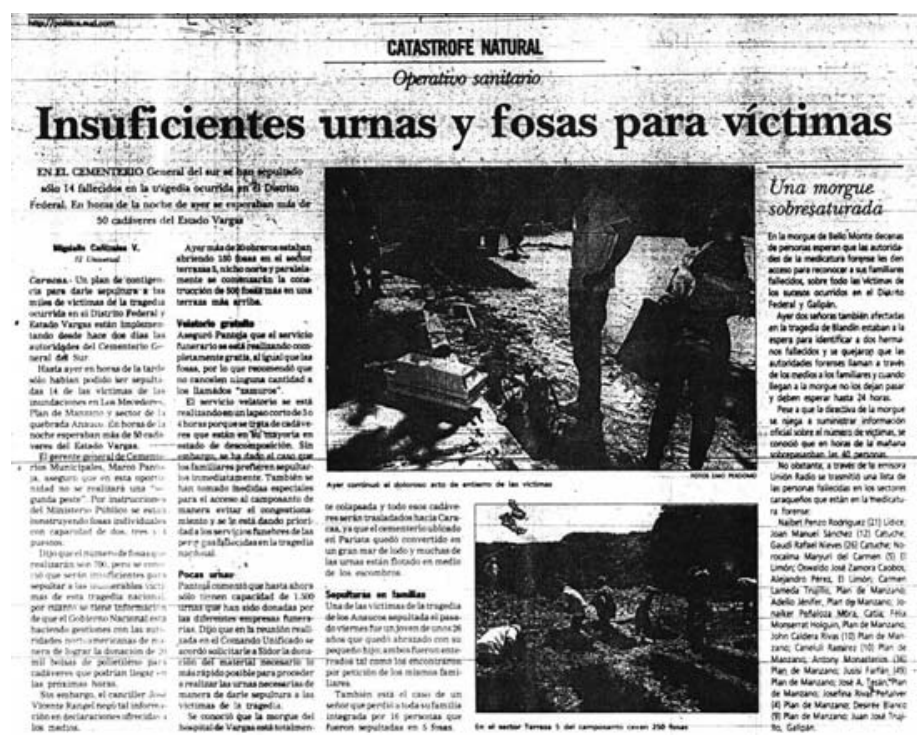

Figura 4: Titular del diario El Universal del 19 de diciembre de 1999.

Dentro de esos números destacan las observaciones acerca del probable desconocimiento de la cifra final, al lado de la mención a los "sepultados" por los aludes. Con esto, la idea de que los "desaparecidos" resultaba un número mayor al de los cuerpos hallados comenzaba a labrar su propio espacio dentro de la imaginación colectiva y de la representación social del evento. Ciertamente, el nivel de destrucción material impactaba la mirada de todo aquel que contempló la escena. No es imputable a los inexpertos el haber calculado un número al respecto sin contar con las herramientas apropiadas para ello; no obstante, cuando el representante de la mayor organización de ayuda humanitaria internacional entra en escena, las cifras parecen asentarse sobre aquellas primeras estimaciones impactadas por la magnitud de la devastación.

El presidente de la Cruz Roja Internacional señaló que las víctimas podían ascender a 50.000, y tal apreciación fue el resultado de un sólo vuelo en helicóptero sobre la zona del desastre. La cifra pareció tan impresionante que ni siquiera fue repetida posteriormente por las autoridades venezolanas, pero bastó, debido al origen de la fuente, para que ocupara varios titulares y para que representara un tope "factible" dentro de aquellas apreciaciones iniciales. Más aún, el hecho de que utilizara como elemento de comparación al huracán Mitch, desastre que cambió substancialmente la forma de comprender la prevención, preparación, mitigación y el concepto de los riesgos y los desastres en América Latina, implicó una rápida colocación del evento entre los más importantes de la historia latinoamericana. 
Esta escena de desconcierto acerca del número de fallecidos, acompañó al propio caos surgido frente a la tragedia. Precisamente, uno de los aspectos que define a un desastre como tal, es el hecho de que todas las respuestas que una sociedad posee ante un evento adverso, se ven superadas. En éste, como en muchos otros casos, la inconsistencia frente a un aspecto tan importante como lo es el trato a las víctimas (fallecidos, damnificados, desplazados y familiares de desaparecidos), resultó ser una evidencia de la mencionada ausencia de respuestas ante el evento, contribuyendo con ello a generar un caos sobre el asunto y a dejar en claro que no fueron utilizadas las herramientas capaces de atender con eficacia a los cuerpos hallados, identificados o no. Los "Manuales y Guías sobre Desastres" [ I I, I2], y en especial las publicaciones sobre el manejo de cadáveres en casos de muertes masivas, publicados por la OPS posteriormente al evento de 1999 en el estado Vargas, dan cuenta literalmente de la necesidad de atender estos aspectos.

\section{OBJETIVOS:}

A través de la revisión (para el caso aquí presentado) de la ausencia de aplicación de las mencionadas técnicas y métodos para el trato de los cadáveres y víctimas rescatados en una tragedia de muertes masivas, esta investigación pretende demostrar una de las consecuencias negativas que a ello sucede. El hecho de que no se cuente hasta el presente con una cifra oficial que estime el número de fallecidos en el desastre de 1999 en el estado Vargas de Venezuela, indica que, por un lado, no se aplicaron (bien por desconocimiento, o bien por negligencia), estrategias de sistematización para el trato posterior al levantamiento de los cuerpos hallados. Sí se aplicaron técnicas forenses básicas en la protocolización de los casos, pero el destino de esa información no trascendió los archivos de las medicaturas.

Al precisar el número de fallecidos, este trabajo pretende asimismo, evidenciar que el manejo inexacto de las cifras declaradas públicamente, ha contribuido a la construcción de una imagen distorsionada del desastre, demostrando con ello, igualmente, el desinterés en corregir las declaraciones iniciales al respecto. La existencia de nuevas técnicas para el manejo de los cadáveres producto de muertes masivas por desastres, ofrece mejores alternativas para el trato a los fallecidos, así como una respuesta más eficaz para los familiares de esas víctimas, toda vez que los resultados de esas sistematizaciones permiten poseer mayor claridad en medio del impacto de un desastre de graves consecuencias. Ciertamente, en diciembre de 1999, Venezuela no contaba con el conocimiento al respecto pues, entre otras razones, algunas de las publicaciones sobre el tema (los mencionados "Manuales y Guías sobre Desastres", por ejemplo), no se habían producido aún. No obstante, la existencia posterior de estos manuales, debería permitir atender en retrospectiva el caso y cerrar definitivamente la situación creada en torno al número de fallecidos en aquella tragedia. Esta investigación intenta aportar soluciones en ese sentido.

\section{MATERIALES Y MÉTODOS:}

Con el objeto de estimar más certeramente un probable número de fallecidos en aquel evento, han sido revisados los estudios previos que al respecto realizaran Altez y Revet [ I3, I 4], en donde se abordaron los archivos de las oficinas forenses que actuaron durante y posteriormente al desastre, así como los cementerios en donde fueron enterrados los cuerpos reconocidos como víctimas de los aludes (Fig. 5). Del mismo modo, se han llevado a cabo entrevistas abiertas (indagaciones no sistematizadas que resultan de una aproximación cualitativa a la región), a familiares de las víctimas y a miembros de la Asociación de Familiares de Personas Extraviadas (agrupados de esta manera a raíz de los hechos), al tiempo que se efectuaron aproximaciones etnográficas a las localidades y comunidades afectadas. 
La información obtenida al concluir esta experiencia, se cruzó también con datos estadísticos de las poblaciones pertenecientes al estado Vargas, a fin de contrastar las cifras emitidas por las autoridades. Esta relación demográfica resultó, posteriormente, un argumento determinante para los razonamientos sobre el caso.

Sobre la base de que quienes actuaron en el rescate de los cadáveres fueron equipos designados por las autoridades nacionales (conformados por forenses, militares y fiscales públicos, por lo general), se estima como cierto que en cada una de estas actuaciones se elaboraron protocolos de casos para ordenar el levantamiento de los cuerpos. En base a estos protocolos, que descansan hoy en los archivos de las medicaturas, muchos de los cuerpos pudieron ser identificados.

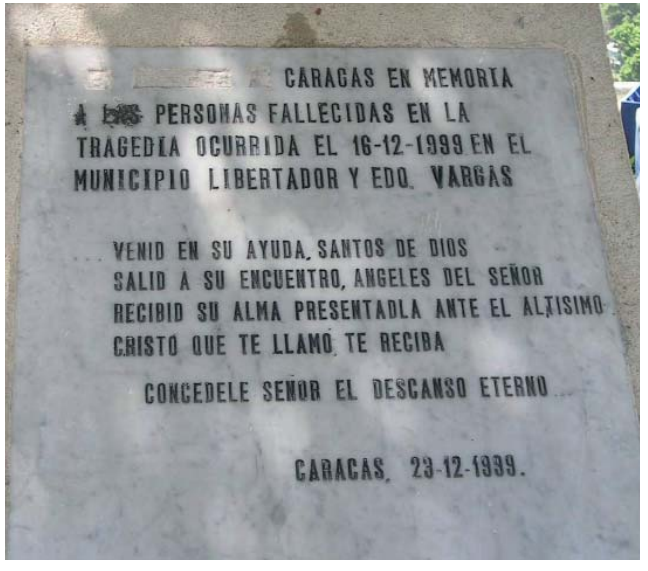

Figura 5: Placa en memoria de los fallecidos en la tragedia del estado Vargas, colocada por las autoridades municipales de Caracas en el Cementerio General del Sur, donde se encuentran enterradas las víctimas del desastre. Fotografía: Sandrine Revet.

En las medicaturas también se encuentran las denuncias de desaparecidos, las cuales no solamente fueron formuladas ante estas instancias, sino que del mismo modo fueron elevadas ante entidades públicas y comisiones destacadas especialmente para ello. La información sobre los desaparecidos también resultó útil, toda vez que fue contrastada con los datos de los cuerpos no identificados.

De esta manera, los protocolos, las denuncias de los desaparecidos, las estadísticas de la población, las cifras declaradas sobre posible número de muertes, los registros de los cementerios y las entrevistas a familiares de víctimas y residentes de la región, se convirtieron en bases de datos para esta investigación. De este cruce de información, se deducen los razonamientos que a continuación se desglosan.

\section{DISCUSIÓN:}

Según las estadísticas [15], hacia diciembre de 1999 en todo el estado Vargas residían 308.303 personas. Este estado consta de ocho parroquias o subdivisiones administrativas; tres de ellas (Macuto, Caraballeda y Naiguatá) resultaron las más afectadas por los aludes, mientras el resto sólo recibió embates menos destructores. Entre las tres parroquias sumaban, hacia 1999, 67.758 habitantes, repartidos de la siguiente manera: Macuto, 13.926; Caraballeda, 35.48I; y Naiguatá, I8.45I.

A finales del año 2000 se realizaron nuevas estadísticas [16], donde los resultados anteriores variaron, evidentemente. El total de habitantes del estado era de 230.566; Macuto contaba con 8.52I personas; Caraballeda, 12.121; y Naiguatá, I I.246. La diferencia es significativa y a simple vista, si se siguen las declaraciones sobre las muertes y los desaparecidos, seguramente se asoma el error de achacar a ello tal diferencia. La Tabla I muestra las cifras antes señaladas.

Con la certeza de que estas tres poblaciones resultaron las más afectadas, y al apreciar que la suma de sus habitantes hacia el año 1999 no superaba las setenta mil personas (67.858 es el total de la sumatoria), parece temerario asegurar que 50.000 fallecidos hubiese sido el número final de víctimas fatales. De haber ocurrido de esta manera, el estado Vargas se habría visto afectado en un 16\% de su población total y estas localidades deberían haber asistido a la desaparición de casi todos sus residentes. Esto, ciertamente, no sucedió así. 
Tabla I: Relación de habitantes en el estado Vargas y en las parroquias más afectadas para el año 1999 y para el año 2000, según estimaciones y estadísticas oficiales, y la diferencia simple entre esas cifras.

\begin{tabular}{|c|c|c|c|}
\hline Entidad & $\begin{array}{c}\text { Población estimada } \\
\text { para el año 1999 }\end{array}$ & $\begin{array}{c}\text { Población censada } \\
\text { en el año 2000 }\end{array}$ & Diferencia simple \\
\hline Estado Vargas (total) & 308.303 & 230.566 & 77.737 \\
\hline Parroquia Macuto & 13.926 & 8.521 & 5.405 \\
\hline Parroquia Caraballeda & 35.481 & 12.121 & 23.360 \\
\hline Parroquia Naiguatá & 18.451 & 11.246 & 7.205 \\
\hline
\end{tabular}

Por otro lado, si ese número supuesto de cinco decenas de víctimas fatales se hubiese repartido entre todas las parroquias del estado, entonces el número de cuerpos hallados y protocolizados por las actuaciones forenses, debería dar cuenta de ello en el señalamiento del lugar donde fueron hallados los cuerpos. Y esto tampoco sucedió de esta manera. Antes de presentar los resultados hallados en los archivos de las medicaturas forenses, otro dato importante se cruza con la información estadística: el Total de familias ubicadas en núcleos de desarrollo local (documento elaborado por el Fondo Único Social, FUS, en el año 2002 [17], organismo que a la sazón fuese destacado para la atención de las familias afectadas por las lluvias de 1999, y que ofrece resultados de estas actuaciones), donde se aprecia que 12.53 I familias fueron reubicadas en asentamientos destinados para este fin. Estadísticamente, el promedio de integrantes por familia en Venezuela fue calculado en 5 personas para las estimaciones de 1999, lo cual supone que si se multiplica el total de familias asistidas luego del desastre por 5, la cifra de 62.655 personas (mínimo), se acerca claramente a la diferencia de poblaciones entre las estimaciones sobre 1999 y el censo del año 2000. Una relación de estas cifras se ofrece en la Tabla II.

Tabla II: Relación entre los totales poblacionales del estado Vargas presentados por estadísticas oficiales y las diferencias entre esos totales luego del desastre, con base en el número de familias reubicadas por el gobierno nacional.

\begin{tabular}{|c|c|c|c|}
\hline $\begin{array}{c}\text { Población estimada } \\
\text { para el año 1999 }\end{array}$ & $\begin{array}{c}\text { Población censada } \\
\text { en el año 2000 }\end{array}$ & Diferencia simple & $\begin{array}{c}\text { Número estimado } \\
\text { de personas } \\
\text { reubicadas luego } \\
\text { del desastre }\end{array}$ \\
\hline 308.303 & 230.566 & 77.737 & 62.655 \\
\hline
\end{tabular}

Estas cifras impiden pensar que la diferencia entre los habitantes del año 1999 y los que residían en la región hacia el año 2000, esté determinada por el fallecimiento de varias decenas de miles de ellos. La primera conclusión a la que se debe llegar es que el número de 50.000 fallecidos es exagerado e impreciso. 
Por otro lado, una resta simple entre las personas reubicadas y la diferencia de habitantes calculada entre los residentes de 1999 y los censados en el 2000, arroja un número de 15.082 personas. Suponer que este sería el estimado de víctimas fatales de la tragedia, es otro error, pues no todos los habitantes del estado Vargas fueron asistidos por el gobierno en la política de reubicación de familias, ni todos los habitantes de la región fueron censados. De esta manera, este número, o bien un aproximado, tampoco resulta un indicador fiable ni un estimado pertinente.

A partir de estas cifras y de la duda razonable acerca del número de muertes, se visitaron los archivos de las Medicaturas Forenses de Caracas y La Guaira (esta última asiste a la región del estado Vargas y recomenzó a trabajar de manera operativa a partir de los últimos meses del año 2000). En la Medicatura de Caracas fue posible encontrar la mayor información posible en relación a la actuación del personal destacado para el levantamiento de cadáveres. Parte de la investigación se concentró en sistematizar los protocolos, clasificando la información obtenida en dos grandes grupos: restos hallados (identificados y no identificados), y desaparecidos (casos sin resolver denunciados por familiares, con la denuncia sostenida hasta por dos años o más). La Tabla III indica los resultados obtenidos, en donde se suman tres números más, los cuales son el resultado de dos testimonios (sumados como "identificados") y un hallazgo (efectuado en el proceso de esta investigación, durante la remoción de escombros en una de las zonas afectadas, sumado como "no identificado", por tratarse solamente de un esqueleto, ver Fig. 6).

Tabla III: Resultados totales de la sistematización de la información hallada en las Medicaturas Forenses.

\begin{tabular}{|l|l|}
\hline Restos hallados & $\mathbf{5 2 1}$ \\
\hline Identificados & 231 \\
\hline No identificados & 290 \\
\hline Desaparecidos & 331 \\
\hline
\end{tabular}

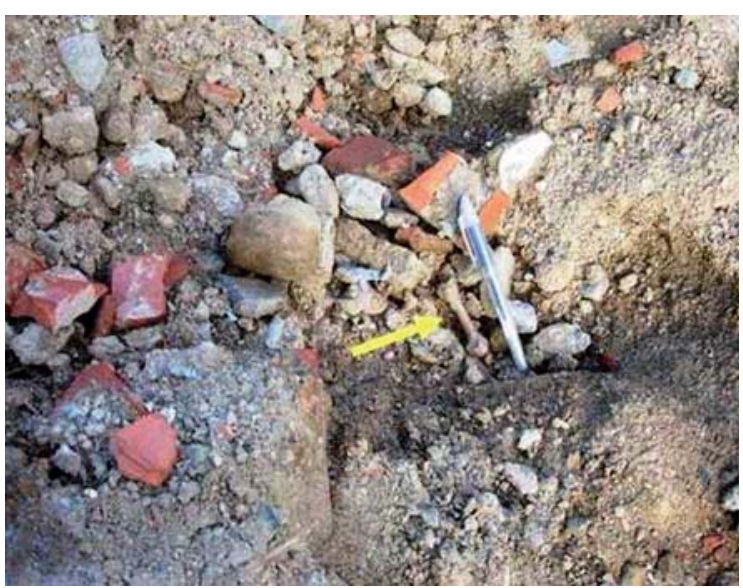

Figura 6: Resto óseo hallado en el proceso de esta investigación, en la localidad de Carmen de Uria, al este del estado Vargas. Fotografía: Rogelio Altez.
Tabla IV: Totales de causas de muerte halladas en los protocolos de las Medicaturas Forenses.

\begin{tabular}{|l|c|}
\hline Causas de muerte & Totales \\
\hline No especificada & 327 \\
\hline $\begin{array}{l}\text { Politraumatismos por } \\
\text { sepultamiento }\end{array}$ & 111 \\
\hline Politraumatismos & 15 \\
\hline Acc. vásculo-cerebral & 1 \\
\hline Asfixia mecánica & 39 \\
\hline Infarto & 7 \\
\hline Insuficiencia respiratoria & 2 \\
\hline TCE severo & 2 \\
\hline $\begin{array}{l}\text { Fallo multiorgánico/ } \\
\text { Shock séptico }\end{array}$ & 2 \\
\hline Cardiopatía dilatada & 1 \\
\hline $\begin{array}{l}\text { Hemorragia digestiva alta/ } \\
\text { Varices esofágicas }\end{array}$ & 2 \\
\hline Sin estudio & \\
\hline
\end{tabular}


Para sistematizar la información, se elaboraron listas en donde se clasificaron los datos de la siguiente manera: $N^{\circ}$ correlativo (correspondiente a la cantidad de casos); $N^{\circ}$ del protocolo; fecha de estudio; nombre de la víctima (en caso de conocerse); edad (estimada o verificada); sexo; causa de muerte (si fue posible determinarse); procedencia del cadáver; sitio de entierro. Esto permitió conocer con más detalles la situación. Por ejemplo, las causas de muerte registradas fueron las siguientes (señaladas así en los protocolos): politraumatismos por sepultamiento; politraumatismos; accidente vásculo-cerebral; asfixia mecánica; infarto; insuficiencia respiratoria; traumatismo cráneo-encefálico severo; fallo multiorgánico/shock séptico; cardiopatía dilatada; hemorragia digestiva alta/várices esofágicas; y causa no especificada. También fue hallado un significativo número de protocolos sin estudio. El resultado de la sistematización de las causas de muertes se enseña en la Tabla IV.

Estos totales presentados en las tablas III y IV, deben acompañarse con aspectos cualitativos de la investigación (entrevistas abiertas y de profundidad) y empíricos (visitas a los cementerios donde han sido enterradas las víctimas del desastre: Cementerio General del Sur, en Caracas, y Cementerio La esperanza, estado Vargas). En ambos casos resultó evidente que las cifras volvían a oscilar entre totales que no lucían con una relación coherente, puesto que, por ejemplo, al preguntar a testigos y familiares por el número de muertes entre sus seres queridos o en su zona de residencia, las respuestas corroboraron lo hallado. Esto debe explicarse de la siguiente manera, pues las preguntas realizadas fueron tres, básicamente, a saber: I) "iCuántas personas fallecieron en su grupo familiar?"; 2) "iCuántas personas fallecieron en su zona de residencia?"; y 3 ) "iCuántas personas cree que fallecieron en el desastre?".

Ante la primera y segunda preguntas, las respuestas mayoritarias fueron negativas (indicando que no hubo muertes entre sus familiares o vecinos), o bien reducidas al número de personas que fallecieron en el círculo inmediato del interrogado, lo cual luce absolutamente coherente con las cifras reducidas halladas en esta investigación. No obstante, ante la tercera pregunta, las respuestas oscilaban entre 15.000 y 25.000 víctimas, con lo cual se corrobora el peso de los discursos oficiales del poder y de los medios de comunicación. De la mano de ambas plataformas discursivas (no siempre compartiendo los mismos objetivos), se construyó la representación social de un elevado número de víctimas con la tragedia de 1999. Sin importar que lo experimentado en el desastre haya conducido a un resultado extremadamente distante a las cifras del poder y de los medios masivos, se ha asumido que el evento de aquel diciembre sepultó decenas de miles de personas, aunque ese número jamás haya sido comprobado ni formara parte de las vivencias de las víctimas.

Al terminar de realizar esta sistematización de la información hallada, es posible asegurar que la sumatoria simple de restos hallados (52I) y desaparecidos (33I), supone un total de 852 personas fallecidas. No obstante, resulta llamativamente significativo el hecho de que el número de cuerpos no identificados (290), sea menor al de los desaparecidos, con lo cual es de suponer que buena parte de los restos sin identificar formen parte de las víctimas que no fueron halladas por sus familiares. Si esto es así, entonces puede concluirse que el total de muertes en el desastre de 1999 no supera las 700 personas. Algo muy distante a las decenas de miles con las que las autoridades y los medios de comunicación lograron construir un escenario fatal que simplemente contribuyó a satisfacer intereses económicos y de poder.

\section{CONCLUSIONES: el problema de la no aplicación de técnicas y metodologías apropiadas para el tratamiento de muertes masivas por desastres.}

Es un hecho históricamente demostrado que las muertes masivas implican problemáticas 
complejas para cualquier sociedad. Antes de la modernidad, en los contextos sociales de las colonias ibéricas en América, por ejemplo, las muertes masivas ocasionadas por desastres (especialmente en el caso de terremotos y epidemias), significaban un grave problema para su enterramiento. Sin las técnicas del presente (desarrolladas en América hacia la segunda mitad del siglo XIX [I8], los cadáveres de los virulentos, por ejemplo, apenas eran sepultados bajo un túmulo de poca profundidad, pues el privilegio de ser enterrados en las iglesias no correspondía a muertes indecentes o factibles de causar contagio (siguiendo la antigua creencia de que los cuerpos continúan contaminando después del deceso), o bien eran entregados a los animales de rapiña en las afueras del poblado, amortajados si era el caso. Los vecinos que tenían la suerte de poseer cal, la utilizaban para calcinar al cadáver, aunque era ésta una práctica de privilegiados. En todo caso, las muertes masivas aportaban problemas que se extendían desde lo práctico hasta lo moral y lo legal. En no pocas situaciones, ante una gran cantidad de fallecidos, las sociedades coloniales o de las tempranas repúblicas decimonónicas, optaban por las piras funerarias (hay ejemplos documentados de esto para el caso del terremoto de 1812 en Caracas [19]), aún cuando tal medida fuese en contra de la fe cristiana y significase una severa paradoja para las gentes de entonces, la cual, finalmente, era resuelta y justificada por el pragmatismo de la emergencia.

Ya hacia finales del siglo XVIII, la situación problemática que representaba el enterramiento de cadáveres virulentos, llevó el caso a manos de la corona. Una Real Cédula de 1787, dictada por el rey Carlos III, ordenó que se construyesen cementerios extramuros y que cesaran los enterramientos en las iglesias, salvo en el caso de los prelados y obispos. Con esto se procuraba preservar la salubridad y adecentar el proceso de enterramiento. La medida se tomó por la incapacidad que de suyo representaban las iglesias en su rol de tierra santa y cementerio, puesto que la mayoría ya habían excedido el espacio destinado para ello. En el documento se menciona el "hedor insoportable", la "insalubridad" y el problema que sobrevenía a ello a causa de las muertes por epidemias, las cuales, para entonces, eran masivas. De este modo, la modernidad comenzaba a pronunciar sus recursos legales para corregir la tradición al respecto y secularizar los ejercicios propios del ritual fúnebre.

Esta breve mención a los antecedentes se hace pertinente, puesto que desde entonces la sociedad moderna no ha desplegado transformaciones jurídicas sustanciales para la práctica de los enterramientos, ni siquiera frente a las graves circunstancias que sobrevienen a las muertes masivas.

En la actualidad, el trato a estos casos genera controversias morales y problemas prácticos, especialmente en países empobrecidos, donde el impacto de los desastres es aún mayor, debido a la falta de estrategias de prevención y atención a emergencias, corrupción en el trato de recursos destinados a ayudas humanitarias, condiciones materiales y sociales vulnerables, y marcos legales no preparados para casos similares. Es por ello que han sido publicados en los últimos años los citados "Manuales y Guías sobre Desastres", en donde se hace mención específica al "manejo de cadáveres", como una necesidad que debe atenderse con cuidado por parte de los Estados en donde han ocurrido desastres con muertes masivas. En estas publicaciones se ha insistido en el "rol crítico para normar y conducir" [I l] el trato a los cadáveres por parte de las autoridades competentes, pues el mismo "tiene un efecto profundo y duradero en la salud mental de los sobrevivientes y de las comunidades" [12]. Estas afirmaciones demuestran que la no aplicación de estrategias que atiendan efectivamente los casos de muertes masivas, producen resultados negativos en todos los niveles de la sociedad, especialmente en las comunidades más afectadas por ello. No obstante, a pesar de la existencia de estos manuales, la aplicación de las técnicas y métodos al respecto no depende de su puesta en práctica luego de la irrupción del desastre, sino de la importancia de contar con estos recursos como planes preventivos, es decir, antes de los hechos. 
Morgan y cols. [8], señalan la existencia de estrategias para el trato a familiares de víctimas fatales en el caso de desastres (especialmente la atención psicológica), al tiempo que observan la carencia de técnicas para el trato, propiamente, de esas muertes masivas. Entre los problemas resultantes de fallecimientos numerosos, destacan los siguientes: ausencia de sitios (predestinados al respecto) apropiados para los enterramientos masivos; falta de técnicas para la identificación de cadáveres a medio plazo; inexistencia de marcos jurídicos que atiendan la problemática; y la carencia de herramientas que sistematicen la información sobre el número de muertes en forma de respuesta rápida. Todos estos aspectos se observaron como consecuencia del desastre de 1999 en el estado Vargas.

Con el caso del tsunami del sur asiático en diciembre de 2004, las muertes masivas desnudaron los problemas antes enumerados, agravando la situación cuando los conflictos morales ocuparon la escena. En los lugares más afectados, Tailandia: 8.345 personas fallecidas, Sri Lanka: 35.399, e Indonesia: 165.708 [8], las respuestas ante la situación evidenciaron la ausencia de técnicas y preparación: fosas comunes sin tratamiento ni metodologías, cremación masiva y enterramientos por grupos sin identificar, son algunas de las características de la situación. Los mismos autores señalan que estas reacciones fueron el resultado del temor común a los cadáveres. Con estos casos se demuestra que los antiguos miedos a la contaminación y a la posibilidad de que se desate una epidemia después de que un desastre cause fallecimientos numerosos, todavía se mantienen intactos, a pesar de más de dos siglos de llustración, modernidad y ciencia aplicada. Entre los miedos y la carencia de técnicas, los desastres profundizan sus alcances especialmente en el trato a las muertes masivas.

Perera [7] y Morgan y cols. [8], propusieron estrategias que resuelven esta problemática, a través del despliegue de técnicas y metodologías que podrían atender la situación. Siendo los objetivos más importantes el rescate de los cuerpos y la identificación, en ambos casos deben desplegarse recursos humanos y materiales que logren el objetivo. La recuperación de cadáveres debe realizarse sistemáticamente, puesto que es éste el primer paso de la identificación, toda vez que, más tarde, cuando los cuerpos sean tratados por los forenses, los datos vinculados al levantamiento de los fallecidos acompañen a la información médica que se desprende del proceso de identificación. El trato a estos cuerpos requiere, además, de recursos no siempre disponibles en el momento de la emergencia.

En efecto, los autores sugieren contar con equipos que puedan refrigerar los cuerpos mientras se atienden, para evitar la rápida descomposición de los mismos. No debe olvidarse que en el caso del sur asiático, las muertes se elevaron fácilmente por encima de las decenas de miles, lo cual dificultó la atención de los expertos, pues se trataba de un número difícil de manejar (en algunos casos se recurrió a la utilización de hielo seco). Asimismo, los cadáveres deben estar acompañados de la información suministrada por los forenses y por la recogida en el proceso de su levantamiento, para que más tarde todo ello contribuya a su identificación definitiva. Después de esto, y contando con la información ya sistematizada y disponible públicamente, se procede a los "enterramientos masivos" (mass burials grounds), en localidades previamente seleccionadas al efecto, de manera que ello facilite el tratamiento a los cadáveres y la accesibilidad posterior para la posible identificación a manos de familiares o dolientes, a través de la exhumación.

Uno de los aspectos más delicados de esta propuesta descansa en el problema ético y moral que supone la idea de una "fosa común" para numerosos cadáveres. La representación más característica de los enterramientos masivos es, precisamente, la imagen negativa de la fosa común, pues ésta se asocia con recuerdos dramáticos de las guerras mundiales, o bien con el trato inhuma- 
no y la supresión de todos los derechos que se producen en las dictaduras. No obstante, los mass burials grounds a los que hacen referencia los autores citados, deben ser el resultado de una perspectiva especialmente opuesta a estas representaciones, pues en realidad parte de la consideración a las víctimas y del respeto al derecho que todo doliente posee de hallar a su ser querido. La carga moral negativa que surge de la idea de una fosa común, resulta comprensible si se atiende la representación que de ello se tiene; sin embargo, en este caso se hace énfasis en las necesidades que sobrevienen a las muertes masivas por desastres, y el trato que merecen las víctimas (mortales y sobrevivientes) a tales casos. Esto implica un despliegue técnico y metodológico que se apoya en recursos médicos, forenses, antropológicos y legales, para solventar crisis que por lo general superan las capacidades de respuestas de las sociedades afectadas.

Ciertamente, esta propuesta requiere, en especial, de gobiernos organizados y responsables, así como de sociedades preparadas para responder acertadamente ante amenazas que puedan causar muertes masivas. En el caso del desastre de Vargas en 1999, es importante señalar que se contó (tal como ya se observó), con la designación de comisiones oficiales para el levantamiento de los cadáveres, así como con la identificación primaria de los cuerpos a manos de los forenses; no obstante, la información nunca se hizo pública; la sistematización de los datos no se homologó con el objeto de cruzar información; se solaparon esfuerzos (entre las distintas instituciones que asistieron la emergencia); se confundió a la opinión pública con discursos desinformados e interesados solamente en los escenarios políticos; se llevaron a cabo enterramientos sin regularizar la información; y, finalmente, nunca se dio un trato realmente interesado al caso de los desaparecidos, contribuyendo con ello a la confusión general.

Toda esta problemática formó parte del desastre, no como una consecuencia, sino como parte estructurante del mismo. De hecho, y como un perfil característico de la situación, en las entrevistas realizadas a la Asociación de Familiares de Personas Extraviadas, fue posible detectar el trato confuso y superficial por parte de autoridades policiales e instituciones públicas al caso de los desaparecidos. Una buena parte de estos casos representaban la búsqueda de niñas, niños y adolescentes cuyos padres o representantes aseguraban que se encontraban con vida, pues ellos y varios testigos daban por cierto que fueron rescatados con vida por helicópteros del ejército nacional en el área de desastre, o bien separados de sus familiares en medio de las labores de asistencia y emergencia, las cuales también evidenciaron la inmensa confusión y falta de preparación al respecto.

De las 331 denuncias de desaparecidos, 120 pertenecen a menores de 18 años (se cuentan sólo los que tenían hasta 17 años inclusive), y entre ellas se encuentran 20 casos bajo sospecha de vida; es decir, veinte de los menores de la lista de desaparecidos cuentan con pruebas de vida (débiles o sustentables), recogidas por sus familiares y entregadas a las autoridades competentes, las cuales no pasaron de ser registradas en expedientes que jamás contaron con la debida prosecución. Más conmovedor resulta el testimonio directo de algunos padres que relatan cómo vieron por última vez a sus hijos cuando fueron rescatados por helicópteros frente a sus ojos, mientras ellos mismos ayudaron a que los chicos se subiesen; o bien otros casos donde los familiares cuentan cómo sus menores desaparecieron en los aeropuertos de Maiquetía, Valencia o La Carlota (Caracas), justo cuando los helicópteros descargaban a los rescatados, o en medio del caos en el que estaban envueltos esos aeropuertos ante la llegada de las personas trasladadas desde los lugares del desastre.

En buena medida, estas situaciones (con consecuencias siempre dramáticas), son el resultado de la falta de prevención y preparación para la atención a los desastres. Generalmente, lo que luce como prioritario en el proceso anterior a los eventos adversos, descansa en las medidas de asistencia y emergencia (cuerpos de rescate, movilización militar, designación de centros de acopio, 
nombramiento de comisiones interinstitucionales, etc.), pero no en el desarrollo de herramientas que den respuesta a la problemática que sobreviene a las muertes masivas. Uno de los aspectos que suman problemáticas y trastornos a estos casos, se observa en la compleja figura de los desaparecidos, puesto que siempre son tomados como "posibles muertes", al tiempo que la atención que estas situaciones reciben usualmente no cuentan con recursos sistemáticos que den respuestas eficaces, o bien que separen a ambas instancias y les traten como casos distintos. Los desastres continúan desnudando el subdesarrollo cada vez que un contexto vulnerable sucumbe ante una amenaza.

El caso del desastre del estado Vargas, Venezuela, en diciembre de 1999, representa un ejemplo claro de la problemática que se desprende de la ausencia de técnicas, métodos y preparación para el trato de muertes masivas sobrevenidas como consecuencia a un evento adverso. La disposición privilegiadamente política sobre el despliegue posterior al desastre, contribuyó a que los discursos al respecto distorsionaran la realidad y confundieran a la opinión pública nacional e internacional sobre el caso. Esto generó una idea errónea sobre el número de muertes en el evento, así como una situación crítica en el trato de las víctimas que todavía en la actualidad no ofrece soluciones al respecto.

Tras realizar esta investigación, en donde ha sido posible sistematizar la información sobre el problema, se ha llegado a la conclusión de que el número de muertes en aquella tragedia no debe superar a los 700 fallecidos. Sin embargo, han resultado mucho más eficaces los discursos políticos y mediáticos, toda vez que juntos (aunque con intereses encontrados), construyeron una plataforma interpretativa a través de la cual la sociedad se representó la tragedia como un desastre que causó decenas de miles de muertes. Esa misma forma de abordar la situación (a partir de intereses políticos y económicos), impidió que se manejaran cifras oficiales y definitivas de manera pública, generando más dudas y confusiones sobre el asunto.

Con esta investigación no se espera convencer a las comunidades afectadas en el desastre de 1999 acerca de cuál es el "verdadero" número de víctimas fatales en aquel evento. Si la representación social generada por los discursos públicos desplegados al efecto (en donde la idea de que los fallecidos rondan cifras que oscilan entre los 15.000 y los 50.000), contribuye a que la sociedad genere memoria colectiva y mecanismos de defensa consecuentes al respecto, parece más pertinente asistir a la construcción de procesos sociales en los que las comunidades solidifican sus respuestas frente a eventos adversos, preparando a generaciones posteriores a través del desarrollo de su memoria. Es ésta una paradoja sin solución, pues la representación casi mítica del desastre, quizás aporte herramientas culturales con las que enfrentar eventos similares en el futuro.

Tras revisar con cuidado el caso expuesto en esta investigación, es posible concluir, asimismo, que el papel de la medicina forense en estas situaciones resulta fundamental. No se trata solamente de la intervención en el proceso del rescate de cadáveres o de los esfuerzos especializados para la identificación de los mismos; los forenses poseen en sus manos la posibilidad de generar información sistematizada y responsable sobre el número de víctimas y la relación entre cuerpos identificados y sin identificar, lo cual es, hasta el presente, un problema no siempre resuelto luego de que irrumpe un desastre de muertes masivas. Asumir este papel como parte del perfil de la medicina forense, fortalecería la práctica social de la ciencia al respecto, y contribuiría a reducir las vulnerabilidades características de las sociedades en riesgo, pues al aumentar la capacidad de respuesta frente a la posibilidad de un desastre, se previenen las consecuencias negativas de estos eventos, garantizando respuestas exitosas en medio de las crisis y las adversidades. 


\section{AGRADECIMIENTOS:}

El autor quiere expresar su gratitud con los colegas antropólogos Rosario Massimo, Ana Luisa López, Lourdes Pérez, Juan Rodríguez, Maryorit Pacheco, Maritza Garaicoechea, Livia Muñoz y Moravia Lozada, de la Escuela de Antropología de la Universidad Central de Venezuela y de la Medicatura Forense de Caracas, así como a los señores Alicia Ocando de Liendo y David Paz, sobrevivientes de la tragedia y familiares de personas desaparecidas. Igualmente, se hace extensivo el agradecimiento a los revisores y árbitros del trabajo en Cuadernos de Medicina Forense, quienes contribuyeron con sus observaciones a mejorar la calidad de esta investigación.

\section{BIBLIOGRAFÍA:}

I.- CEPAL-PNUD: Los efectos socioeconómicos de las inundaciones y deslizamientos en Venezuela en 1999, Sede subregional de la Comisión Económica para América Latina y el Caribe (CEPAL), México, D. F. 2000. Documento inédito.

2.- Maskrey, A. (comp.): Los desastres no son naturales. Red de Estudios Sociales en Prevención de Desastres en América Latina (LA RED). Bogotá. 1993.

3.- Altez, R.: Desastres y conocimiento; breve ensayo sobre la mirada histórica de la sismología. En: J. Á. Rodríguez, (comp.). Visiones del oficio. Historiadores venezolanos en el siglo XXI. Academia Nacional de la Historia-Comisión de Estudios de Postgrado-Facultad de Humanidades, UCV, Caracas, 2000. pp. 453-474.

4.- García Acosta, V.: El riesgo como construcción social y la construcción social del riesgo. Desacatos, Revista de Antropología Social, CIESAS, México, 2005, septiembre-diciembre, 19: II-24.

5.- Altez, R.: Historia sin memoria: la cotidiana recurrencia de eventos desastrosos en el estado Vargas, Venezuela. Rev. Geog. Venezolana, Mérida. 2005; Vol. Esp.: 313-342.

6.- Autoridad Única de Área para el Estado vargas (AUAEV): Plan de Ordenamiento del Área de Protección y Recuperación Ambiental del Eje Arrecife - Los Caracas. Noviembre, 200I. Documento inédito.

7.- Perera, C.: After the Tsunami: Legal Implications of Mass Burials of Unidentified Victims in Sri Lanka, Plos Medicine, June 2005, Vol. 2 (6): el85, 3 p.

8.- Morgan, O. W.; P. Sribanditmongkol; C. Perera; Y. Sulasmi; D. Van Alphen; E. Sondorp: Mass Fatality Management following the South Asian Tsunami Disaster: Case Studies in Thailand, Indonesia, and Sri Lanka. Plos Medicine, June 2006, Vol. 3 (6): el95, 7 p.

9.- Declaraciones del entonces Director de Defensa Civil, Ángel Rangel, al periódico venezolano El Universal, de fechas 17 de diciembre de 1999 (pág. 4-17) y 22 de diciembre de 1999 (pág. I-4).
10.- Declaraciones de George Weber al periódico El Universal en fecha 22 de diciembre de 1999 (pág. I-II).

II.- Organización Panamericana de la Salud (OPS): Manejo de cadáveres en situaciones de desastres. Serie Manuales y Guías sobre Desastres, № 5, Washington, 2004.

12.- Organización Panamericana de la Salud (OPS), Organización Mundial de la Salud (OMS), Comité Internacional de la Cruz Roja (CICR), Federación Internacional de Sociedades de la Cruz Roja y de la Media Luna Roja: La gestión de cadáveres en situaciones de desastre, guía práctica para equipos de respuesta. Serie Manuales y Guías sobre Desastres, № 6, Washington, 2006.

13.- Altez, R. y Revet, S.: Contar los muertos para contar la muerte: Discusión en torno al número de fallecidos en la tragedia de 1999 en el estado Vargas. Rev. Geog. Venezolana, Mérida. 2005; Vol. Esp.: 2l-43. I4.- Altez, R., Revet, S.: Certezas y paradojas: discusión en torno al número de fallecidos en la tragedia de 1999 en el estado Vargas. Bol. Hist. Geoc., Caracas. 2004; 94:21-26.

15.- Oficina Central de Estadística e Informática (OCEI): Proyecciones para 1999. Bol. Estadístico, Caracas. 1997.

16.- Oficina Central de Estadística e Informática (OCEI): Censo de estructuras, viviendas y personas en las zonas afectadas del estado Vargas. Caracas. 2000.

17.- Fondo Único Social (FUS): Total de familias en los núcleos de desarrollo local. Caracas, 20 de febrero de 2002. Documento inédito.

18.- Núnez, M.: La muerte secularizada. Estudio sobre las transformaciones en los modos de enterramiento en la Provincia de Caracas: 1787-1876. Tesis de grado, Escuela de Antropología, UCV, Caracas. 2004.

19.- Altez, R.: El desastre de 1812 en Venezuela: sismos, vulnerabilidades y una patria no tan boba. Universidad Católica Andrés BelloFundación Empresas Polar, Caracas. 2006. 